\title{
Im Kommunikationszeitalter dem Menschen dienen
}

\author{
von André Ruszkowski
}

Bei einer Aussage über den Beitrag der Kirche zur Kommunikation der Zukunft sehe ich die Hauptschwierigkeit in der Vorausschau der Kommunikationsentwicklung überhaupt.

Die Generation, der ich angehöre, erlebte die höchst aufsehenerregende „Kommunikationsexplosion" der Geschichte. Das Kino, Ende des vergangenen Jahrhunderts kaum erfunden, überschwemmte die am meisten zurüickgebliebenen Regionen mit Filmen, und das in weniger als dreißig Jahren. Der Rundfunk erlebte einen ähnlichen Aufschwung sofort nach dem Ersten Weltkrieg. Ihm folgte das Fernsehen nach dem Zweiten Weltkrieg. Die Schallplatte, in Millionen Exemplaren verkauft, rundet das Bild. Eine unübersehbare Anzahl von Familien ist heute ausgerüstet, daheim Bild und Ton zu empfangen, die - entweder "direkt" oder "gespeichert" von allen vier Enden der Erde kommen. Neben diesen modernen Mitteln, die vornehmlich auf elektronischem Weg arbeiten, nimmt sich die gedruckte Presse bisweilen wie ein armer Verwandter aus, sei es auch nur vom Standpunkt der Schnelligkeit der Information aus.

Will man jedoch diese aufsehenerregende Erfahrung auf Zukunft hin projizieren, drängen sich zwei Feststellungen auf:

Die erste betrifft das Mißverhältnis zwischen dem überwältigenden Fortschritt der Technologie einerseits und der oft enttäuschenden Qualität der „Botschaften“, die diese Technologie übermittelt. Wir verfügen über hervorragende Werkzeuge, aber was machen wir daraus?

Es ist nicht die Zeit, hier die soziologischen, wirtschaftlichen und politischen Gründe $\mathrm{zu}$ analysieren, die zusammentreffen, die von den "Massenmedien“ angebotenen Kulturprodukte so mittelmäßig machen und zur Passivität ihrer Kundschaft beisteuern. Ein überreiches Schrifttum besteht schon zu diesem Thema. Aber man darf sich fragen, ob dieses Mißverhältnis eine Art historischer Schicksalhaftigkeit darstellt und sich von heute bis zum Jahr 2000 noch verschärft.

Die zweite Feststellung wird vielleicht helfen, die Antwort auf diese Frage zu finden. Niemand kann leugnen, daß eine Beziehung besteht zwischen der Qualität der Produktion und den Produktionsstrukturen. Man müßte also, um die demnächst anstehende Entwicklung einer Aktivität vorauszusehen - in diesem Fall geht es um die "Massenmedien“ - , den wahrscheinlichen Wandel ihrer Strukturen kennen. Nun stehen wir hier jedoch hinsichtlich der Strukturen der "Massenmedien “ einer großen Unbekannten gegenüber.

Es ist noch nicht lange her, da gestattete alles, eine immer stärkere Konzentration der großen "Massenmedien“ in den Händen einer abnehmenden Anzahl von -

Prof. Dr. André Ruszkowski ist emeritierter Direktor des „Institut des Communications Sociales" an der St. Pauls-Universität in Ottawa. 
öffentlichen oder privaten - Personen oder Unternehmungen vorauszusehen. Angesichts einiger weniger mächtiger Inhaber der Kommunikationsmittel wäre dann die Masse des Publikums verurteilt, mehr oder weniger passiv, mehr oder weniger beeinflußbar deren Kommunikation zu konsumieren.

Eine solche Entwicklung der Strukturen böte in Sicht auf die künftige Qualität der Produktionen kaum große Hoffnung. Selbst wenn man annähme, daß die Herren der Medien sich der Mühe unterzögen, diese zu vervollkommnen, wäre es doch wenig wahrscheinlich, daß sie nicht auch weiterhin vor allem ihre Privatinteressen verfolgten. Das Publikum könnte sich zwar organisieren, um einen Druck auszuüben, aber es hätte nicht das letzte Wort.

Im Lauf der letzten Jahre entwickelt sich die Technologie allerdings in eine ganz andere Richtung fort. Neben dem "schweren" und kostspieligen Material, das nur den Professionellen und den einigermaßen bedeutsamen Unternehmen zugänglich ist, sieht man neue Instrumente aufkommen, die weniger teuer und leichter sind und für Amateure wie Einzelpersonen oder Gruppen mit begrenzten Mitteln verfügbar sind. Ohne die Tendenz zur Konzentration zu verdrängen, wird eine neue Tendenz möglich. Sie könnte den Einsatz der modernen Kommunikationsmittel dezentralisieren, da sie einer immer größeren Anzahl von Einzelpersonen und Gruppen ermöglicht, sich auf diese Weise in der Gesellschaft verständlich zu machen.

Beide Feststellungen - jene des Mißverhältnisses $\mathrm{zwischen} \mathrm{dem} \mathrm{technologischen}$ Fortschritt und der Qualität der Botschaften, und jene andere der Tendenzen zur Zentralisation und zur Dezentralisation, die, wenn nicht parallel, so zumindest gleichzeitig verlaufen - erlauben die Aussage, daß die Kommunikation des Jahres 2000 den Menschen noch beängstigender herausfordert, als sie es heute schon tut.

Es wird also darum gehen, die Kommunikationsmittel, die immer vielfältiger und vollkommener werden, zu einem echt humanistischen Ziel einzusetzen, das heißt zum Dienst an der menschlichen Person. Und das in einer doppelten Richtung:

Einmal dahin, die Kreativität zu fördern und so einer größeren Anzahl von Einzelmenschen zu ermöglichen, mit ihresgleichen den Reichtum ihres inneren Lebens zu teilen. Sodann dahin, die Medienerfahrung als wichtiges und konstruktives Element des Weltverständnisses für jeden Einzelmenschen zu integrieren, dessen Persönlichkeit durch die kritische und einsichtige Aufnahme der von den Medien übermittelten Botschaften bereichert werden soll.

Eine solche Sicht führt uns dahin, eine erste Vorstellung über den möglichen Beitrag der Kirche zu entwickeln.

Angesichts der Herausforderungen der Medien erfährt der heutige Mensch - und das trifft vermutlich noch stärker im Jahr $2000 \mathrm{zu}$ - eine tiefe Angst. Der geballte Ablauf von Ideen, Informationen, Bildern und Tönen brandet über ihn hinweg. Wie soll man es anstellen, um sich nicht erdrücken zu lassen? Wie soll man seine Persönlichkeit wahren? Wie soll man sich nicht zugunsten von Interessen manipulieren lassen, die bisweilen den eigenen entgegengesetzt sind? Was soll man tun, um dieses Uberbranden $z \mathfrak{u}$ beherrschen und es in den Dienst seiner eigenen Weiterentwicklung $\mathrm{zu}$ stellen?

Nicht das heutige Erziehungssystem und noch weniger das überkommene Lebensmilieu werden die Antworten auf solche Fragen liefern. 
Man könnte die Situation vergleichen mit der des primitiven Menschen, den Analphabetentum und Unwissen daran hindern, in der Erkenntnis fortzuschreiten. Diesen Menschen zu lehren, unternahm die Kirche als Aufgabe. Um ihn fähig zu machen, die religiöse Unterweisung aufzunehmen, begann sie, ihm Unterweisung schlechthin anzubieten. Sie stellte sich in seinen Dienst, um ihm zu helfen, seine Persönlichkeit zu entwickeln.

Mir scheint die Menschheit zum heutigen Zeitpunkt einen analogen Dienst seitens der Kirche zu erwarten, damit sie (kulturell wie auch in geistlicher Hinsicht) die Erfahrung der Massenmedien besser verkraftet.

Wenn die Kirche all ihre intellektuellen und institutionellen Hilfen mobilisiert, um die Potentiale der sozialen Kommunikationsmittel für den Dienst an der menschlichen Person zu entdecken und um sie dem Publikum unterschiedslos zur Verfügung zu stellen, dann wird sie ihrer Sendung und ihrer schönsten Tradition als Kulturträger treu sein. Sie wird dem Menschen von heute helfen, eine der schwersten Krisen seiner Epoche zu überwinden.

In dem Maße, in dem die öffentliche Meinung die Sorge der Kirche wahrnimmt, diese Hilfe für alle zu leisten, wird sie ein wirkliches Interesse an den Stellungnahmen und Lehren der kirchlichen Autoritäten entwickeln. Denn sie wird deren Wert nicht nur in spezifisch religiöser, sondern auch in schlicht menschlicher Hinsicht erkennen.

Das bedeutet natürlich nicht, daß man sich den kulturellen und sozialen Sorgen zuwendet und darüber die unmittelbare Aufgabe der Evangelisation vernachlässigt, für die die Kirche an erster Stelle verantwortlich bleibt. Es bedeutet nur, daß es in diesem Bereich ebenso wie auch in anderen dem Menschen als Gottes Geschöpf Dienste zu erweisen gilt, ohne welche die Evangelisation im strengen Sinn weder aufrichtig noch wirksam wäre.

Im übrigen wird man erst dann, wenn man die Glaubwürdigkeit der Kirche durch die Qualität ihrer Lehren und ihrer praktischen Taten erwiesen hat, positive Ergebnisse mit den zur Evangelisation bestimmten Medien erzielen, vornehmlich im „audiovisuellen “ Bereich.

Die Aufgabe der katholischen Universitäten und anderer Forschungs- und Lehrzentren, wie sie die Pastoralinstruktion "Communio et Progressio" vorsieht, ist niemals dringlicher gewesen. Sie sollen Kenntnis des Kommunikationsphänomens in den christlichen Kreisen vertiefen und verbreiten.

Wieviele innere und äußere Schwierigkeiten der Kirche hätten vermieden oder vermindert werden können, wenn unser interner Kommunikationsaustausch besser funktionierte und wir es verständen, ein echteres und ein zugleich auf das öffentliche Empfinden besser eingestelltes Image nach außen hin zu projizieren!

Inwiefern hätte nicht das ganze Zeichen- und Symbolsystem, das die Kirche bereits zu einer von der Kommunikationsgeschichte gezeichneten Zeit entwickelte, die Chance, sich mit neuen Elementen anzureichern, die durch die neuen Techniken möglich geworden sind?

Die grundlegende Erforschung all dieser Perspektiven wird unabdingbar vorausgesetzt, damit die Kirche wesentlich zur Lösung der Probleme beitragen könnte, die die Kommunikation schon jetzt stellt und noch mehr im Jahr 2000 stellen wird. 
Sie kann auch den inneren Wandel der Kirche mitbestimmen, dessen Rhythmus seit dem Zweiten Vatikanum immer stärker spürbar wird.

\section{S U M M A R Y}

In this century human society has experienced a communications explosion of the mass Inedia. There is a disproportion between the progress of technology and the quality of the messages. We are still uncertain about the structures of mass media. The disproportion and the tendencies both towards centralization and de-centralization of communications will occupy the growing concern of man in the future. How can man preserve his individuality and not become controlled by the media? This problem shows the contribution the Church can make. She has to identify the real values not only in "a religious but also in a human way. With her duty to evangelize she has to contribute also towards the humanizing of communications. Thus she can contribute also towards a solution of these problems in the future.

\section{RESUMÉ}

L'histoire assista, au cours de ce siècle, à une "explosion de la communication « par les "mass media«. Il y a une disproportion entre le progrès de la technologie et la qualité des "messages«. Pour ce qui est de la structure des "mass media «, nous nous trouvons cependant face à une inconnue. La dite disproportion et les tendances, aussi bien à la centralisation qu'à la décentralisation de la communication, provoque, à l'avenir, l'homme de façon encore plus inquiètante. Comment l'homme doit-il conserver sa personnalité et ne pas se laisser dominer par les mass media? Dans la maîtrise de cette tâche repose aussi le devoir de l'Eglise: ne pas seulement désigner les valeurs véritables comme purement religieuses, mais aussi comme humaines. A côté de la tâche d'évangélisation, elle peut aussi contribuer, par l'»humanisation« de la communication, essentiellement à la solution des problèmes d'avenir.

\section{RE S U M E N}

La Historia vivió en este siglo una "explosión comunicadora “ de los »medios de comunicación«. Existe desproporción entre el progreso de la tecnología y la calidad del "mensaje». Con respecto a las estructuras de los »medios de comunicación « nos encontramos ante un desconocido. La citada desproporción y las tendencias tanto a la centralización como a la descentralización de la comunicación retan en el futuro al hombre de modo más alarmante. Cómo puede mantener el hombre su personalidad sin dejarse dominar por los medios? La misión de la Iglesia incluye también la consecución de este objetivo: dar a conocer no solo los valores meramente religiosos sino también los humanos. Junto a la misión evangelizadora, la Iglesia puede contribuir substancialmente a la solución del problema mediante la shumanización « de la comunicación. 